\title{
Self-Raman Nd:YVO4 laser and electro-optic technology for space-based sodium lidar instrument
}

\author{
Michael A. Krainak, Anthony W. Yu, Diego Janches, \\ Sarah L. Jones, Branimir Blagojevic, Jeffrey Chen \\ NASA Goddard Space Flight Center, Greenbelt, MD, USA 20771
}

\begin{abstract}
We are developing a laser and electro-optic technology to remotely measure Sodium ( $\mathrm{Na}$ ) by adapting existing lidar technology with space flight heritage. The developed instrumentation will serve as the core for the planning of an Heliophysics mission targeted to study the composition and dynamics of Earth's mesosphere based on a spaceborne lidar that will measure the mesospheric Na layer. We present performance results from our diode-pumped tunable Q-switched self-Raman c-cut Nd:YVO4 laser with intra-cavity frequency doubling that produces multi-watt $589 \mathrm{~nm}$ wavelength output. The c-cut Nd:YVO4 laser has a fundamental wavelength that is tunable from 1063-1067 nm. A CW External Cavity diode laser is used as a injection seeder to provide single-frequency grating tunable output around $1066 \mathrm{~nm}$. The injection-seeded self-Raman shifted Nd:VO4 laser is tuned across the sodium vapor D2 line at $589 \mathrm{~nm}$. We will review technologies that provide strong leverage for the sodium lidar laser system with strong heritage from the Ice Cloud and Land Elevation Satellite-2 (ICESat-2) Advanced Topographic Laser Altimeter System (ATLAS). These include a space-qualified frequency-doubled 9W @ 532 nm wavelength Nd:YVO4 laser, a tandem interference filter temperature-stabilized fused-silica-etalon receiver and high-bandwidth photon-counting detectors.
\end{abstract}

Keywords: Sodium lidar, Nd:YVO4 laser, space-based science instruments, optical filters

\section{EARTH MESOSPHERIC SCIENCE}

In recent years, remote-sensing satellites have obtained the first global characterization of the basic structure of the Mesosphere and Lower Thermosphere (MLT) region in terms of large-scale temperature and wind climatologies, resulting in a much richer picture of the structure and variability of the mesosphere. Although these measurements have shown the high temporal variability of both the zonal mean state as well as large scale organized perturbations, such as planetary waves and atmospheric tides, they failed at providing information required for the fundamental characterization of how the basic state is established and maintained.

Layers of neutral metal atoms, such as Iron (Fe), Magnesium $(\mathrm{Mg})$, Calcium $(\mathrm{Ca})$, Potassium $(\mathrm{K})$ and Sodium $(\mathrm{Na})$, which peak between 85 and $95 \mathrm{~km}$ and are $\sim 20 \mathrm{~km}$ in width, are produced by the daily ablation of billions of Interplanetary Dust Particles (IDPs). As these metallic species are ionized during ablation, by sunlight's ultraviolet photons, or by charge exchange with existing atmospheric ions, meteoroids affect the structure, chemistry, dynamics, and energetics of the Mesosphere and Lower Thermosphere (MLT). The strong optical signals that some of these metal layers produce, in particular the Na layer, provides information on the composition, temperature and winds of the MLT making them an optimal tracer of atmospheric dynamics and circulation and potentially enabling the measurement of quantities that are critical to address several compelling scientific questions related to the Earth's Upper Atmosphere and the Geospace Environment. Specifically, we know that Gravity waves (GWs) having wavelengths smaller than a few hundred $\mathrm{km}$ are the dominant contributors to momentum transport and deposition in the MLT, which largely drive the global circulation and thermal structure and interactions with the tides and planetary waves in this region. However, predicting their impact on atmospheric chemistry and dynamics is a major uncertainty in current atmospheric models. Thus there is a pressing need in the ITM community to be able to perform high-resolution measurements that can be used to characterize these waves and their effects in the MLT on a global basis. Such measurements must include highly resolved, in space and time, global temperature and wind profiles, as well as the global distribution of GWs (e.g different orographic forcing sources) and their spectra, which will add to the understanding of key indicators of radiative cooling in the mesosphere. 
Our goal is to develop a spaceborne remote sensing technique that will enable acquisition of global Na density, temperature and wind measurements in the MLT with the spatial and temporal resolution required to resolve issues associated with the structure, chemistry, dynamics, and energetics of this region. Over four decades of ground-based and over two of airborne $\mathrm{Na}$ and Fe lidar observations ${ }^{1,2,3,4}$ have demonstrated that this technique is ideal to obtain highresolution measurements of these quantities. Unfortunately, while these lidars provide very high temporal and vertical resolutions measurements, the ground-based sites are geographically sparse and cannot provide representative global climatologies. Recent models of the mesospheric Na layer ${ }^{5}$ have shown large variations in the sodium constituents over timescales from days to months. These studies also demonstrated that measuring the Na layer at a global scale can enable the study of how external sources impact this region. For example, short-term response of the upper atmosphere to a stratospheric sudden warming is clearly revealed in the sodium column. Seasonality of sodium constituents is strongly affected by variations $s^{6,7}$ in the Meteor Input Function (MIF) and transport via the mean meridional wind. Thus, it is clear that global high-resolution measurements capabilities of the $\mathrm{Na}$ layer using resonance fluorescence lidar will permit, not only measurements of zonal mean structures, planetary waves and tides, but also uniquely characterization of the global distribution of GWs. Such measuring capabilities will enable more accurate constrain of Global Circulation Models (GCMs) via GW drag parameterization enabled by the global estimation GW forcing and transport. Additionally, measuring the global distribution and variability of meteoric $\mathrm{Na}$ that will enable to constrain models of the MLT chemistry, meteoroid ablation and mass loss processes, and Zodiacal Dust Cloud as well as their impact in planetary atmospheres ${ }^{8}$.

A zenith pointing $\mathrm{Na}$ Doppler lidar would provide measurements of vertical profiles of absolute temperature, absolute $\mathrm{Na}$ density and vertical wind perturbations. These data would enable the global characterization of the mesopause region temperature and $\mathrm{Na}$ density structure as well as gravity wave variances and spectra. In addition it would be possible to characterize wave-induced vertical transport of $\mathrm{Na}$ and other mesopause region species by computing the vertical $\mathrm{Na}$ flux profiles and perhaps the heat flux profiles ${ }^{9}$. Vertical constituent transport by gravity waves plays a crucial role in mesopause region chemistry which establishes the structure and seasonal variations of key species such as odd-oxygen. By restricting wind observations to the vertical wind perturbation profiles, rather than absolute winds, the nadir pointing accuracy and stability of the instrument can be relaxed. A more sophisticated scenario is one that will have two beams pointing off zenith in an orthogonal configuration and one beam pointing zenith so that temperature, vector wind and Na density can be measured simultaneously and globally with very high resolution and accuracy. This would further enhance the scientific benefits of the experiment, including the direct measurements of gravity wave momentum flux.

\section{SODIUM LIDAR DEVELOPMENT PATH}

Our science goal is to measure range-resolved atmospheric-sodium-temperature profiles from Low-Earth-Orbit (LEO) using a space-based lidar. The atmospheric temperature is deduced from the linewidth of the resonant fluorescence from the atomic sodium vapor D2 line as measured by a tunable laser as shown in Fig. 1.

As noted earlier, tunable pulsed ground-based $\mathrm{Na}$ lidar systems have provided some mesospheric temperature and wind profiles. However, the key components of the present ground-based sodium lidar systems are not readily transferred to a space-based sodium lidar. Fortunately, a large number of the sodium lidar components are similar to those developed under the NASA Earth Science ICESat-1/GLAS, ICESat-2/ATLAS and pre-Phase-A ASCENDS Instrument Incubator Program missions. Our thesis is that this leverage and experience can provide a quick path to a space-based sodium lidar science instrument.

\subsection{Sodium lidar laser transmitter}

The key sodium lidar component that requires some development and integration into a lidar system demonstration is the laser transmitter. 


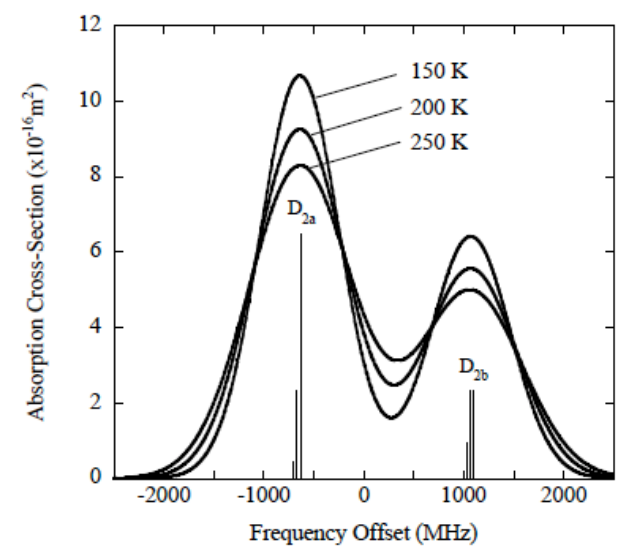

Fig. 1. The D2 resonance line is a Doppler broadened doublet composed of six hyperfine lines of atomic sodium at $589.159 \mathrm{~nm}$ wavelength. The Doppler broadening of the lines is a function of temperature and the ratio of the D2a peak to the value at the minimum between the peaks is a very sensitive function of temperature. (from Reference 10.)

Under a NASA-GSFC IRAD, we conducted a trade study on various approaches for laser development to measure mesospheric sodium. The laser requirements are listed in Table 1.

\begin{tabular}{|l|l|}
\hline Wavelength (nm) & $589.2 \mathrm{~nm} \pm 0.3 \mathrm{~nm}$ \\
\hline Optical Power (@589 nm) & $9 \mathrm{~W}$ \\
\hline Pulsed & Yes \\
\hline Wall-plug efficiency & $>2 \%$ \\
\hline Pulse width & $1-50 \mathrm{~ns}$ \\
\hline Spectral width & $<100 \mathrm{MHz}$ \\
\hline Tuning range & $0.5 \mathrm{~nm}$ \\
\hline Tuning speed & $<10 \mathrm{microseconds} \mathrm{per} \mathrm{GHz}$ \\
\hline Beam Quality (M^2) & $<4$ \\
\hline Spaceflight heritage & Yes \\
\hline
\end{tabular}

Two approaches allow some leverage from previous space-qualification work, 1) mixing the output of two Nd:YAG lasers - one at $1064 \mathrm{~nm}$ and one at $1310 \mathrm{~nm}$; 2) A frequency-doubled self-Raman shifted Nd:YVO4 laser ${ }^{11}$. The ICESat-2/ATLAS mission has a fully space-qualified frequency-doubled $\mathrm{Nd}: \mathrm{YVO}_{4}$ laser transmitter ${ }^{12}$ with 9 Watts of output at $532 \mathrm{~nm}$ (Figure 2). Since the self-Raman shifted $\mathrm{Nd}: \mathrm{YVO}_{4}$ laser only requires one laser that is very similar to the ICESat-2 ATLAS laser with very few non-space-qualified components - we selected it over the two Nd:YAG laser approach. A diagram of our sodium lidar laser approach is shown in Figure 3. We use a c-cut $\mathrm{Nd}: \mathrm{YVO}_{4}$ crystal to produce tunable laser light from $1062.2-1066.7 \mathrm{~nm}$ (rather than the a-cut which lases at $1064 \mathrm{~nm}$ ). A typical c-cut $\mathrm{Nd}: \mathrm{YVO}_{4}$ tuning curve ${ }^{13}$ is shown in Figure 4. An active Q-switch is used the to produce high-peak-power pulses to exploit a non-linear self-Raman shift to $1178 \mathrm{~nm}$ wavelength in the exact same crystal. The Q-switching provides the time-of-flight pulses for range-resolved atmospheric temperature measurements while also increasing the intra-cavity frequency-doubling efficiency for $589 \mathrm{~nm}$ wavelength output generation. A tunable injection seeding $1066 \mathrm{~nm}$ wavelength diode laser allows us to tune the $\mathrm{Nd}: \mathrm{YVO}_{4}$ laser to (and across) the exact sodium $\mathrm{D} 2$ line. The injection seeding allows rapid all-electronic wavelength tuning. 


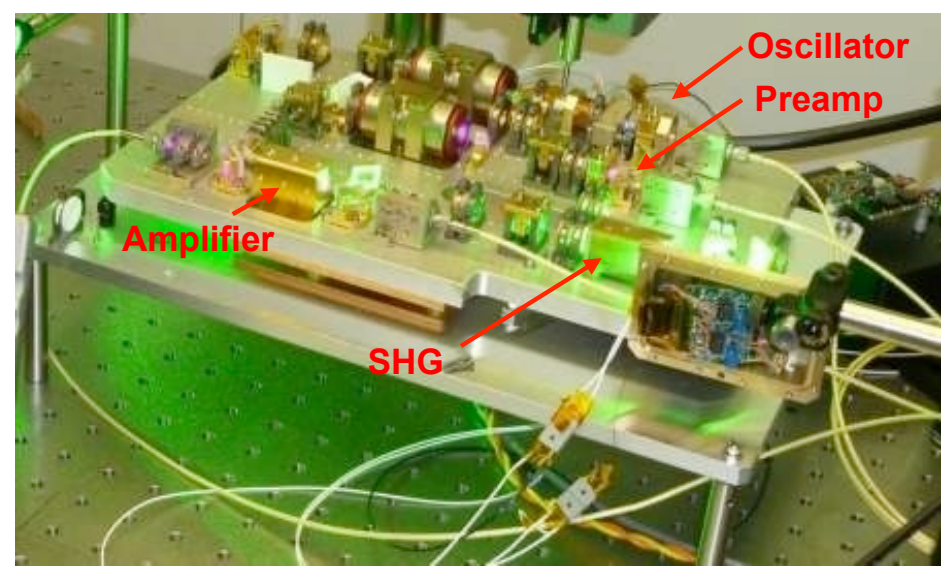

Fig. 2. Brassboard ICESat-2/ATLAS $9 \mathrm{~W}$ Nd:YVO4 laser transmitter (courtesy of Fibertek Inc.)

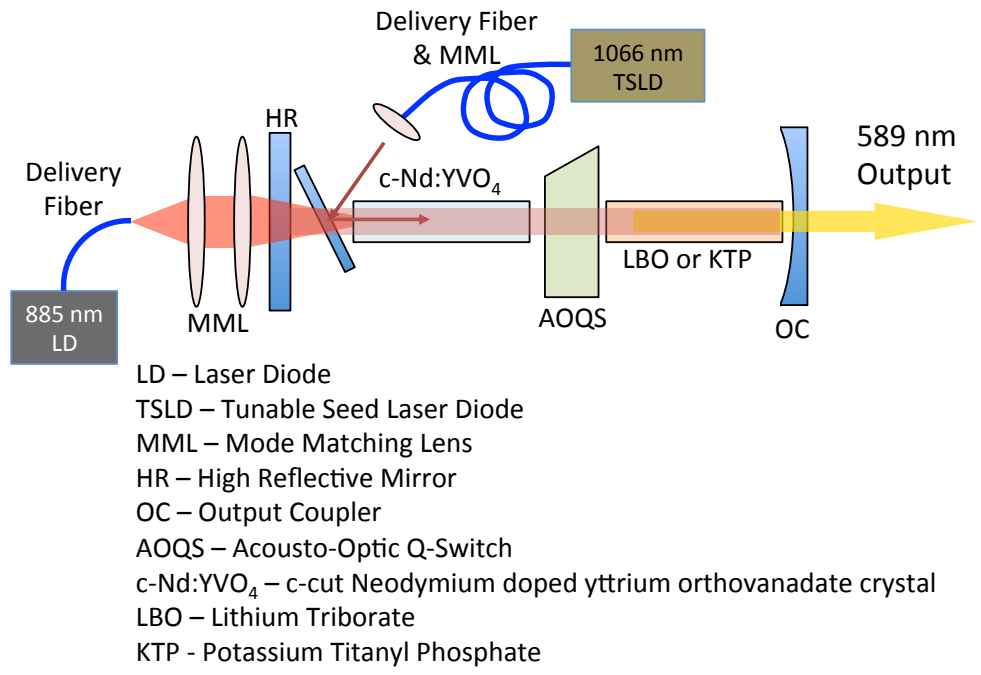

Fig. 3. Sodium lidar injection-seeded $\mathrm{Nd}: \mathrm{YVO}_{4}$ laser transmitter diagram

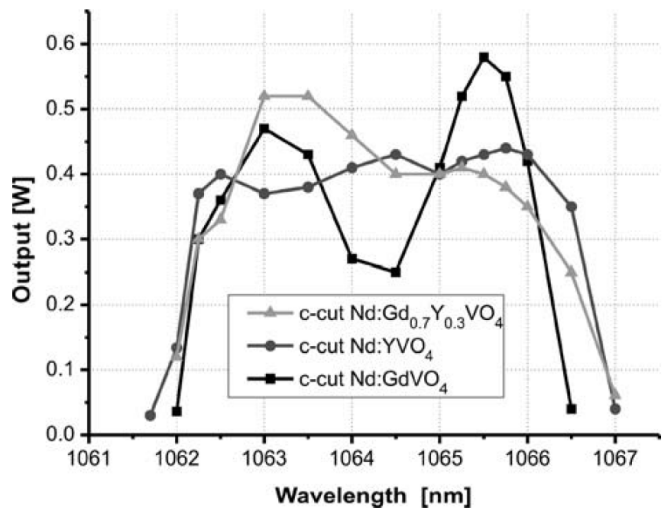

Fig. 4. Wavelength tuning curve for $\mathrm{Nd}^{\mathrm{Y}} \mathrm{YVO}_{4}$ (and two related) laser transmitters (Ref. Sirotkin) 
Referring to Figure 1, the tuning range needed to profile the entire sodium fluorescence spectrum is $4 \mathrm{GHz}$. The equivalent wavelength range at $589 \mathrm{~nm}$ for this $4 \mathrm{GHz}$ frequency range is $4.6 \mathrm{pm}$. We will wavelength lock the injection seed laser to an absolute wavelength to within $4 \mathrm{MHz}$ of the center of the $\mathrm{D} 2$ lines, then using injection current we can tune the laser over the $\pm 2 \mathrm{GHz}( \pm 2.3 \mathrm{pm})$ range to profile the sodium spectrum.

We have constructed a breadboard Self-Raman $\mathrm{Nd}: \mathrm{YVO}_{4}$ laser (Figure 5). To date, we have achieved $\sim 0.5$ Watts of optical power output at $589.4 \mathrm{~nm}$ wavelength with 15 Watts of diode pump power. For this proposed effort, we will demonstrate $9 \mathrm{~W}$ of $589 \mathrm{~nm}$ output that is needed for spaceborne application.

Our laser breadboard is based on c-cut $\mathrm{Nd}: \mathrm{YVO}_{4}$ emitting at $1066.6 \mathrm{~nm}$ then self Raman shifted to $1178 \mathrm{~nm}$ and intracavity frequency doubled to $589 \mathrm{~nm}$. The laser transmitter breadboard is shown in Figure 4, the laser is pumped by a fiber coupled $808 \mathrm{~nm}$ laser diode. This is used to end pump a c-cut $0.3 \%$ atomic-weight $\mathrm{Nd}$ doped vanadate gain medium and a dimension of $3 \mathrm{~mm} \times 3 \mathrm{~mm} \times 20 \mathrm{~mm}$.

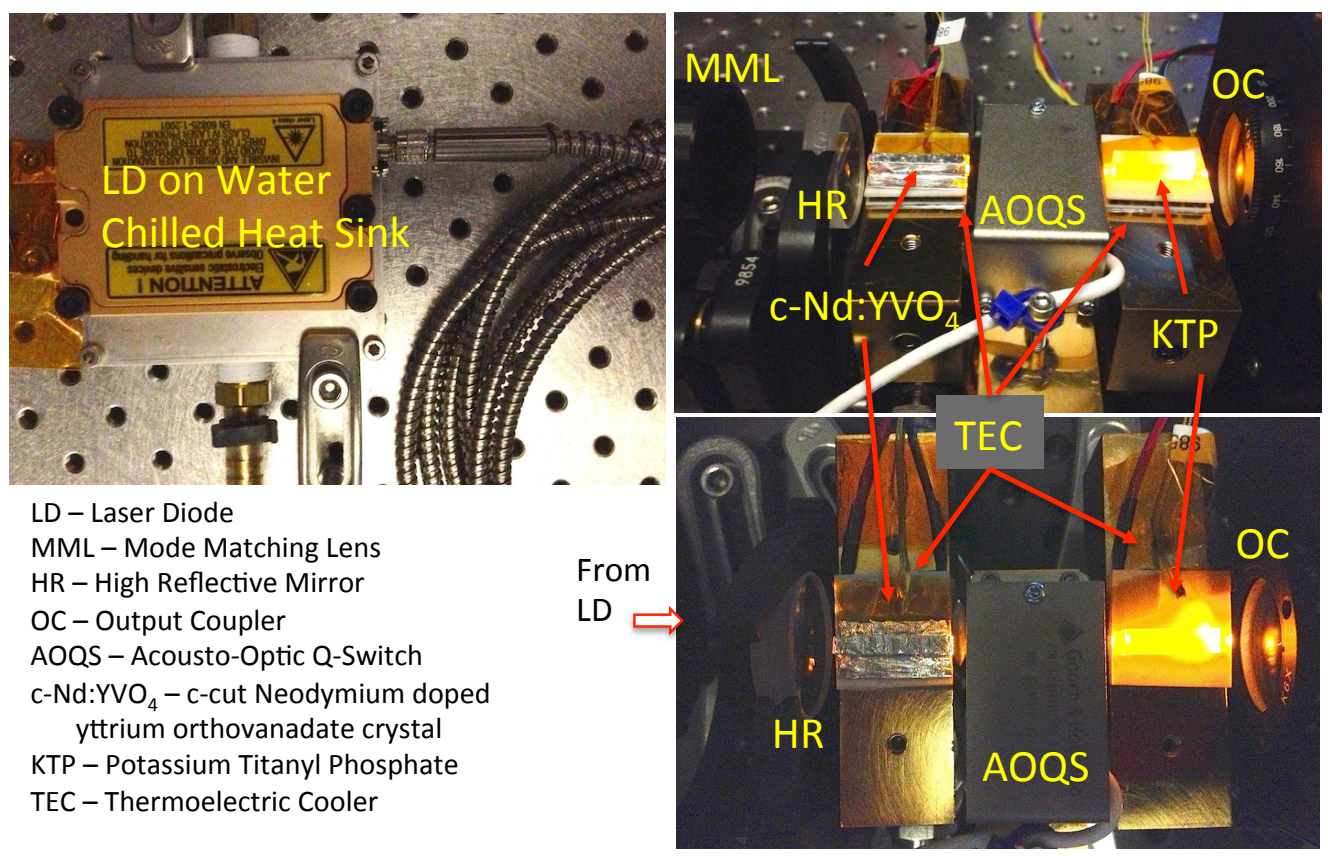

Fig. 5. NASA-GSFC breadboard Self-Raman Q-switched Nd:YVO4 frequency-doubled laser with $589 \mathrm{~nm}$ output.

The laser crystal is wrapped with an indium foil and bonded to a thermoelectric cooler (TEC) for efficient cooling and thermal management. A thermistor located on the side of the laser crystal is used to measure the crystal surface temperature and provide feedback to the temperature feedback loop to maintain the laser crystal to $20^{\circ} \mathrm{C}$. An acoustooptic active Q-switch (AOQS) is used to produce high peak power pulses that enables us to use the self-Raman effect in the exact same crystal to produce a laser at $1178 \mathrm{~nm}$ and enhances the frequency-doubling efficiency to $589 \mathrm{~nm}$. A Lithium Triborate (LBO) or Potassium Titanyl Phosphate (KTP) crystal is used for intracavity frequency doubling. The $\mathrm{KTP}$ is also bonded to a TEC for temperature control for type II critical phase matching at $300 \mathrm{~K}$. In Figure 6 , we show the spectra of our laser breadboard output. The $1066 \mathrm{~nm}$ and the $1178 \mathrm{~nm}$ leak out of the laser cavity and traveled collinearly with the $589 \mathrm{~nm}$ output beam. 


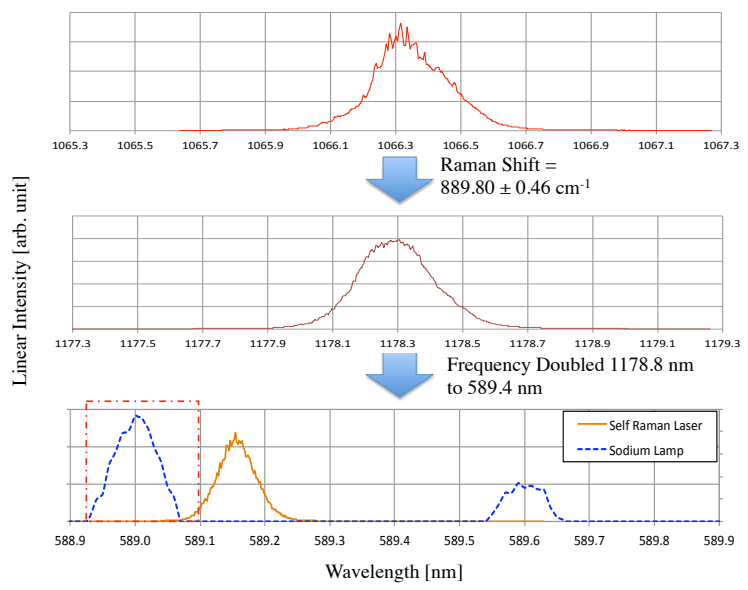

Fig. 6. Measured optical spectra of the NASA-GSFC self-Raman, intracavity frequency-doubled laser breadboard with (not yet tunable) output at $589.4 \mathrm{~nm}$ superimposed with the measured spectra of our sodium vapor lamp. We will optimize the lidar system for the boxed sodium D2 line centered at $\sim 582.2 \mathrm{~nm}$.

\subsection{Laser Wavelength - Locking for Atomic (or Molecular) Spectroscopy}

To tune the wavelength of the Nd:YVO4 laser to the sodium D2 line, we will use injection seed with a tunable Distributed FeedBack (DFB) laser diode at $1066.55 \mathrm{~nm}$. As shown in Fig. 7, we have demonstrated (Numata et al., 2012) a precision and fast wavelength tuning technology that allows a wavelength-locked laser to rapidly scan across an atomic/molecular (e.g. $\mathrm{CO} 2$ or $\mathrm{Na}$ ) absorption line at a user-selectable number of precise wavelengths for remote atmospheric spectroscopy measurements. Our approach uses a master laser that is wavelength-locked to the atomic (or molecular) line center by using a frequency modulation technique, limiting its frequency drift (one standard deviation) to $60 \mathrm{kHz}$ at 0.8 -sec averaging time over 72 hours (Numata et al., 2011).

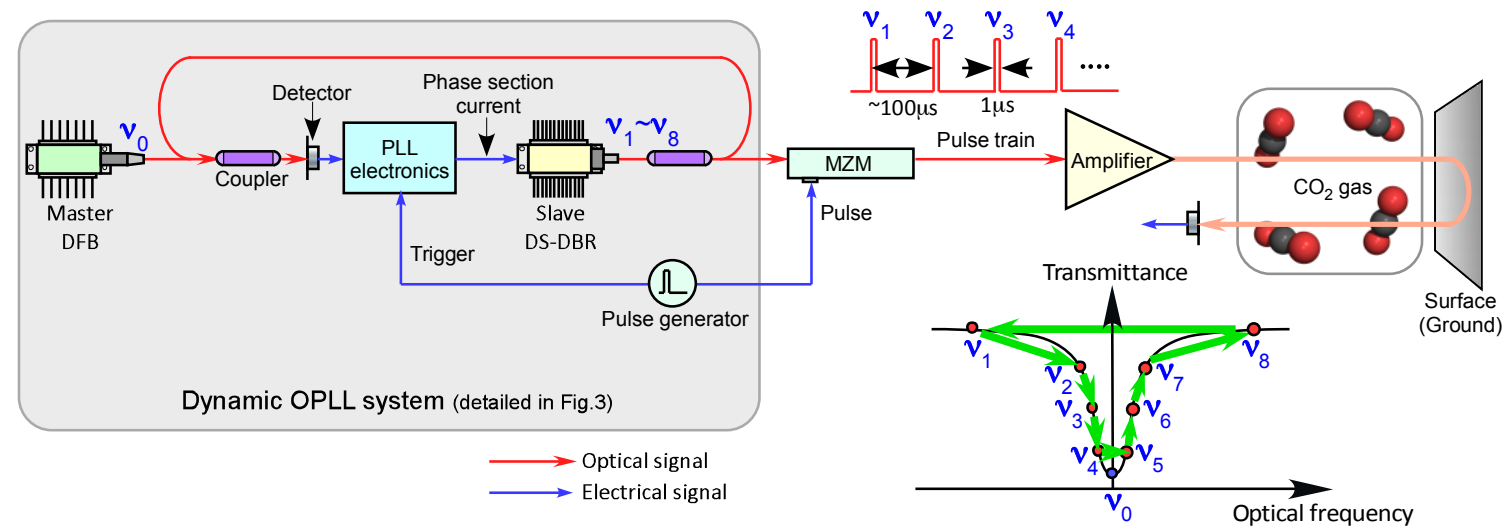

Fig. 7. Concept of the precision fast laser tuning technology and its application for $\mathrm{CO} 2$ (or Na) remote sensing. The slave laser is dynamically offset-locked to the master DFB-LD using an OPLL. The wavelength- stepped pulse train is formed by external modulation through the MZM and subsequent amplification. The amplified pulse train is used to repeatedly measure at multiple points across the $\mathrm{CO} 2$ (or Na) absorption line.

A second (a.k.a. slave) laser diode is dynamically offset-locked to the master laser diode by using an optical phaselocked loop (OPLL), enabling precision fast-tuning of the slave laser to and from any frequencies within a $40-\mathrm{GHz}$ tuning range. For sodium spectroscopy, the master laser is $1178.4 \mathrm{~nm}$ that is doubled to $589.2 \mathrm{~nm}$ and wavelengthlocked to a sodium vapor cell. The offset-locked slave laser is the tunable $1066.55 \mathrm{~nm}$ injection seeder. The OPLL suppresses any slow drift of the offset frequency, allowing the slave laser to retain the absolute frequency stability of the 
master laser. The frequency-stepped pulse train is formed by external modulation through a Mach-Zehnder modulator (MZM) and subsequent amplification. Our technology satisfies stringent requirements for atmospheric gas sensing lidars and enables other applications that require such well-controlled precision fast tuning.

A patent application has been filed for this technology by NASA-GSFC.

\subsection{Ultra-Narrow-Band Optical Filter}

We developed space-qualified ultra-narrow $(28 \mathrm{pm})$ optical filters3 for both the ICESat-1/GLAS and ICESt-2/ATLAS missions and a derivative similar filter was used on the CALIPSO mission. An atomic sodium Faraday filter (Yong et al., 2011) may provide economic and performance benefits over the etalon filter. We are studying the use of both etalon and atomic sodium filters with 5-10 pm optical bandwidth to enable some daytime sodium lidar observations.

\subsection{Detector}

Our space-flight-precursor photon-counting detector technology ${ }^{14}$ is a 16 -channel Photomultiplier Tube with receiver electronics that has been space-qualified for the ICESat-2/ATLAS mission. Our technique uses the 16-channels as a photon-number-resolving "single" detector to provide the required full-spectroscopic sodium lineshape waveform for recovering Mesospheric temperature profiles. We have direct experience using photon-counting receivers for recovering high signal-to-noise ratio single line spectroscopic profiles from our ground ${ }^{15}$ and airborne ${ }^{16}$ pre-ASCENDS mission efforts. Under the proposed effort we will conduct trade studies with the commercial $40 \%$ quantum-efficiency Hamamatsu linear-mode hybrid photomultiplier and new multi-channel silicon avalanche photodiodes similar to those space-qualified by u $^{17}$ on the ICESat-1/GLAS mission. These newer alternate detectors may provide economic and performance benefits over the PMT.

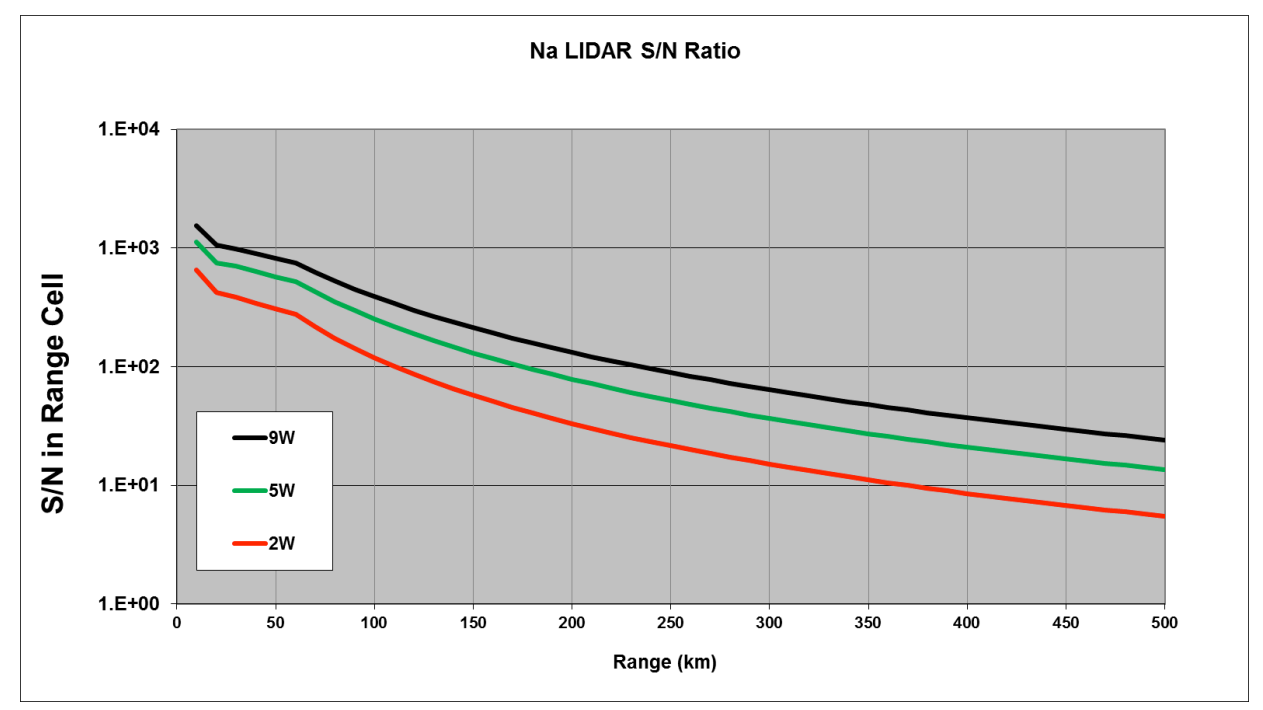

Fig. 8. Sodium Lidar $\mathrm{S} / \mathrm{N}$ ratios for $1 \mathrm{kHz}$ laser pulse rate and the optical background spectral irradiance of $263 \mathrm{~W} / \mathrm{m} 2 / \mu \mathrm{m}$ of averaged Earth albedo (0.31) vs. Sun at 45 degrees.

\subsection{Link Analysis}

Several studies have been conducted analyzing component and performance requirements for space-based sodium lidar ${ }^{4}$. The numerical estimation of the optical power, telescope aperture, detection system and spectroscopic temperature recovery performance is sometimes referred to in the lidar community as a link analysis - borrowing the term from optical communication links. Our own link analysis has been consistently verified by numerous past ground, airborne and space lidar missions. Our preliminary link analysis for a space-based sodium lidar is shown in Figure 8 and Table 2. With a space-based 9 Watt laser optical output at $589.2 \mathrm{~nm}$, a 0.8 -meter optical receiver telescope and a photon-counting receiver, we estimate that we can retrieve global mesospheric temperature profiles with the required temporal $(\sim 30 \mathrm{~s})$, 
altitude $(\sim 1-2 \mathrm{~km})$, temperature $(\sim 1 \mathrm{~K})$ and wind velocity $(\sim 1-5 \mathrm{~m} / \mathrm{s})$ resolution to achieve unprecedented MLT science. Our third-year operational space-flight- precursor ground-based sodium lidar will allow us to directly verify our lidar link analysis by comparing to experimental results to greatly improve extrapolation to a moving Low-Earth-Orbit space-based platform.

\begin{tabular}{|c|c|c|}
\hline $\begin{array}{l}9 \text { W Laser Pulse } \\
\text { Rate }(\mathrm{Hz})\end{array}$ & $\begin{array}{l}\text { Nominal } \\
\text { Operational } \\
\text { Scenario: } \mathrm{S} / \mathrm{N} \text { for } \\
263 \mathrm{~W} / \mathrm{m} 2 / \mu \mathrm{m} \\
\text { optical background }\end{array}$ & $\begin{array}{l}\text { Worst Case } \\
\text { Operational } \\
\text { Scenario: } \mathrm{S} / \mathrm{N} \text { for } \\
1000 \mathrm{~W} / \mathrm{m} 2 / \mu \mathrm{m} \\
\text { optical background }\end{array}$ \\
\hline 1 & 125 & 123 \\
\hline 10 & 119 & 106 \\
\hline 100 & 87.8 & 56.3 \\
\hline 1000 & 37.1 & 19.7 \\
\hline 10000 & 12.9 & 6.3 \\
\hline
\end{tabular}

Table 2: Predicted Signal to Noise ratios $(\mathrm{S} / \mathrm{N})$ of the spaceborne Na-lidar equipped with $9 \mathrm{~W}, 589$ nm pulse operated laser and $0.8 \mathrm{~m}$ receiver telescope with $0.1 \mathrm{mrad}$ field of view, $100 \mathrm{~m}$ range resolution and $10 \mathrm{~s}$ time integration under nominal and worse case optical backgrounds equivalent to 263 and $1000 \mathrm{~W} / \mathrm{m} 2 / \mu \mathrm{m}$ respectively.

\section{REFERENCES}

1. Gardner, C.S. "Introduction to ALOHA-90 - The Airborne Lidar and Observations of the Hawaiian Airglow Campaign" Geophys. Res. Lett. 18,1313-1316 doi: 10.1029/91GL01294. (1991).

2. Chu, X., Pan, W., Papen,G.C., Gardner, C. S., and Gelbwachs, J. A. "Fe Boltzmann temperature lidar: design, error analysis, and initial results at the North and South Poles" Applied Opt. 41, 4400-4410 (2002).

3. Chu, X., Papen,G. C.,, "Resonance Fluorescence Lidar for Measurements of the Middle and Upper Atmosphere" Laser Remote Sensing, T. Fujii and T. Fukuchi, 179-432 (2005).

4. Gardner, C. S., and Liu, A. Z., "Seasonal variations of the vertical fluxes of heat and horizontal momentum in the mesopause region at Starfire Optical Range, New Mexico" Journal of Geophysical Research (Atmospheres), 112 (2007).

5. Marsh, D., Janches, D., Plane, J., and Feng, W., "A Global Model of Meteoric Sodium" J. Geophys. Res. (Atmospheres) Under review. (2013).

6. Janches, D., Heinselman, C., Chau, J., Chandran, A., and Woodman, R., "Modeling the global micrometeor input function in the upper atmosphere observed by high power and large aperture radars" J. Geophys. Res., 111 (2006).

7. Fentzke, J. T., and Janches, D., "A semi-empirical model of the contribution from sporadic meteoroid sources on the meteor input function observed at Arecibo" Journal of Geophysical Research (Space Physics), 113(A03304), (2008).

8. Feng, W., Marsh, D., Chipperfield, M., Janches, D., Hoeffner, J., Yi, and Plane, J., "A Global Atmospheric Model of Meteoric Iron" J. Geophys. Res. (Atmospheres), Under review (2013). 
9. Gardner, C. S. and Liu, A. Z., "Wave-induced transport of atmospheric constituents and its effect on the mesospheric Na layer" Journal of Geophysical Research (Atmospheres), 115 (2010).

10. Carlson, C. G., Swenson, G. R., Dragic, P., and Liu, A., "Maui mesosphere and lower thermosphere (MALT) Na lidar enterprise" Proc. of SPIE 5979 ,59790W, (2005).

11. Duan, Y., Zhu, H., Huang, C., Zhang, G., and Wei, Y., "Potential sodium D2 resonance radiation generated by intracavity SHG of a c-cut Nd:YVO4 self-Raman laser" Optics Express Vol. 19, No. 7 pp. 6333 (2011).

12. Hovis, F., "Qualification of Lasers For NASA Space-Based Remote Sensing Missions: Applying Lessons Learned from CALIPSO to ICESat-2" CLEO JW3C.4., (2012).

13. Sirotkin, A.A., Di Labio, L. Zagumennyi, A.I., Zavartsev, Y.D., Kutovoi, S.A. Vlasov, V.I., Lüthy, W., Feurer, T., Onushchenko, A.A., Shcherbakov, I.A., "Mode-locked diode-pumped vanadate lasers operated with PbS quantum dots" Appl. Phys. B, 94 375-379 (2009).

14. Krainak, M. A., Yang, G., Lu, W., Sun, X., "Photon-counting detectors for space-based applications" Proceedings of the SPIE 7780, Paper 77801-J3 (2010).

15. Allan,G., Riris,H., Abshire, J.B., Sun, X., Wilson, E., Burris, J., and Krainak, M. A., "Laser sounder for active remote sensing measurements of CO2 concentrations" IEEE Aerospace Conference Proceedings, Society of PhotoOptical Instrumentation Engineers (SPIE) Conference Series 1, (2008).

16. Abshire, J.B., Riris, H., and Allan, G., "Pulsed airborne lidar measurements of atmospheric CO2 column absorption" Tellus Series B-Chemical and Physical Meteorology, (2010).

17. Sun, X., Krainak, M. A., Abshire, J. B., Spinhirne, J. D., Trottier, C., Davies, M., Dautet, H., Allan, G. R., Lukemire, A. T., , Vandiver, J. C., "Space qualified silicon avalanche photodiode single photon counting modules," Journal of Modern Optics, Vol. 51. No. 9-10 pp. 1333-1350 (2004). 